\title{
DISCOVERY AND ROSSITER-McLAUGHLIN EFFECT OF EXOPLANET KEPLER-8b*
}

Jon M. Jenkins ${ }^{1}$, William J. Borucki ${ }^{2}$, David G. Koch $^{2}$, Geoffrey W. Marcy ${ }^{3}$, William D. Cochran ${ }^{4}$, William F. Welsh ${ }^{5}$, Gibor Basri ${ }^{3}$, Natalie M. Batalha ${ }^{6}$, Lars A. Buchhave ${ }^{7,8}$, Timothy M. Brown ${ }^{9}$, Douglas A. Caldwell ${ }^{1}$, Edward W. Dunham ${ }^{10}$, Michael Endl ${ }^{4}$, Debra A. Fischer ${ }^{11,12}$, Thomas N. Gautier III ${ }^{13}$, John C. Geary ${ }^{7}$, Ronald L. Gilliland ${ }^{14}$, Steve B. Howell ${ }^{15}$, Howard IsaACson ${ }^{3}$, John Asher Johnson ${ }^{16}$, David W. Latham ${ }^{7}$, Jack J. Lissauer ${ }^{2}$, David G. Monet ${ }^{17}$, Jason F. Rowe ${ }^{2,21}$, Dimitar D. Sasselov ${ }^{7}$, Andrew W. Howard ${ }^{3}$, Phillip MacQueen $^{4}$, Jerome A. Orosz ${ }^{5}$, Hema Chandrasekaran ${ }^{1,20}$, Joseph D. Twicken ${ }^{1}$, Stephen T. Bryson ${ }^{2}$, Elisa V. Quintana ${ }^{1}$, Bruce D. Clarke $^{1}, \mathrm{Jie} \mathrm{Li}^{1}$, Christopher Allen $^{18}$, Peter Tenenbaum ${ }^{1}$, HaYley Wu $^{1}$, Søren Meibom $^{7}$, Todd C. Klaus ${ }^{18}$, Christopher K. Middour ${ }^{18}$, Miles T. Cote $^{2}$, Sean McCaulifF $^{18}$, Forrest R. Girouard ${ }^{18}$, Jay P. Gunter ${ }^{18}$, Bill Wohler ${ }^{18}$, JenNifer R. Hall ${ }^{18}$, KhadeEJah Ibrahim ${ }^{18}$, AKM Kamal Uddin ${ }^{18}$, Michael S. Wu ${ }^{19}$, Paresh A. Bhavsar ${ }^{2}$, Jeffrey Van Cleve ${ }^{1}$, David L. Pletcher ${ }^{2}$, Jessie L. Dotson ${ }^{2}$, and Michael R. Hahs ${ }^{2}$

${ }^{1}$ SETI Institute/NASA Ames Research Center, Moffett Field, CA 94035, USA; Jon.Jenkins@ nasa.gov

2 NASA Ames Research Center, Moffett Field, CA 94035, USA

${ }^{3}$ University of California Berkeley, Berkeley, CA 94720, USA

${ }^{4}$ University of Texas, Austin, TX 78712, USA

${ }^{5}$ San Diego State University, San Diego, CA 92182, USA

${ }^{6}$ San Jose State University, San Jose, CA 95192, USA

${ }^{7}$ Harvard-Smithsonian Center for Astrophysics, Cambridge, MA 02138, USA

${ }^{8}$ Niels Bohr Institute, Copenhagen University, DK-2100 Copenhagen, Denmark

${ }^{9}$ Las Cumbres Observatory Global Telescope, Goleta, CA 93117, USA

${ }^{10}$ Lowell Observatory, Flagstaff, AZ 86001, USA

${ }^{11}$ Radcliffe Institute, Cambridge, MA, USA

12 Yale University, New Haven, CT, USA

13 Jet Propulsion Laboratory/California Institute of Technology, Pasadena, CA 91109, USA

${ }^{14}$ Space Telescope Science Institute, Baltimore, MD 21218, USA

15 National Optical Astronomy Observatory, Tucson, AZ 85719, USA

${ }^{16}$ California Institute of Technology, Pasadena, CA 91109, USA

${ }^{17}$ US Naval Observatory, Flagstaff Station, Flagstaff, AZ 86001, USA

18 Orbital Sciences Corporation/NASA Ames Research Center, Moffett Field, CA 94035, USA

${ }^{19}$ Bastion Technologies/NASA Ames Research Center, Moffett Field, CA 94035, USA

Received 2010 February 14; accepted 2010 September 2; published 2010 November 11

\begin{abstract}
We report on the discovery and the Rossiter-McLaughlin (R-M) effect of Kepler-8b, a transiting planet identified by the NASA Kepler Mission. Kepler photometry and Keck-HIRES radial velocities yield the radius and mass of the planet around this F8IV subgiant host star. The planet has a radius $R_{\mathrm{P}}=1.419 R_{\mathrm{J}}$ and a mass $M_{\mathrm{P}}=0.60 M_{\mathrm{J}}$, yielding a density of $0.26 \mathrm{~g} \mathrm{~cm}^{-3}$, one of the lowest planetary densities known. The orbital period is $P=3.523$ days and the orbital semimajor axis is $0.0483_{-0.0012}^{+0.006}$ AU. The star has a large rotational $v \sin i$ of $10.5 \pm 0.7 \mathrm{~km} \mathrm{~s}^{-1}$ and is relatively faint $(V \approx 13.89 \mathrm{mag}$ ); both properties are deleterious to precise Doppler measurements. The velocities are indeed noisy, with scatter of $30 \mathrm{~m} \mathrm{~s}^{-1}$, but exhibit a period and phase that are consistent with those implied by transit photometry. We securely detect the R-M effect, confirming the planet's existence and establishing its orbit as prograde. We measure an inclination between the projected planetary orbital axis and the projected stellar rotation axis of $\lambda=-26.4 \pm 10.1$, indicating a significant inclination of the planetary orbit. R-M measurements of a large sample of transiting planets from Kepler will provide a statistically robust measure of the true distribution of spin-orbit orientations for hot Jupiters around $F$ and early $G$ stars.
\end{abstract}

Key words: radio lines: planetary systems - stars: fundamental parameters - stars: individual (Kepler-8, KIC 6822244, 2MASS 18450914+4227038)

Online-only material: color figures

\section{INTRODUCTION}

To date, 90 "hot Jupiters"-gas giant planets with periods $\leqslant 10$ days-have been detected around Sun-like stars (Torres

\footnotetext{
* Based in part on observations obtained at the W. M. Keck Observatory, which is operated as a scientific partnership between the California Institute of Technology, the University of California, and the National Aeronautics and Space Administration. The Observatory was made possible by the generous financial support of the W. M. Keck Foundation.

${ }^{20}$ Current address: Lawrence Livermore National Laboratory, Livermore, CA 94551, USA.

${ }^{21}$ NASA Postdoctoral Fellow Program.
}

et al. 2008). The front-running formation scenario supposes that these planets did not form where they reside today, close to their host stars, because the inner regions of protoplanetary disks have inadequate surface densities and high temperatures (Lin et al. 1996). Instead, hot Jupiters are presumed to form several astronomical units (AUs) from their host stars, followed by subsequent migration inward to their current locations. One likely migration scenario involves tidal interactions between the planet and a remaining gaseous disk (Lin et al. 1996; Moorhead \& Adams 2008), causing the planet to spiral inward while maintaining a nearly circular orbit that is co-planar 
with the disk. Alternatively, migration may occur by $N$-body gravitational interactions such as planet-planet scattering (Rasio \& Ford 1996; Chatterjee et al. 2008), dynamical relaxation (Papaloizou \& Terquem 2001; Adams \& Laughlin 2003), and Kozai interactions with a distant object, and be damped later by tidal friction (Holman et al. 1997; Fabrycky \& Tremaine 2007; Wu et al. 2007; Nagasawa et al. 2008).

We assume that planets form in protoplanetary disks with the stellar spin and planetary orbital axes aligned. If so, the nearly adiabatic tidal interactions between planets and disks would maintain the alignment (Ward \& Hahn 1994). In contrast, few-body gravitational interactions would typically cause misalignments. Few-body models by Adams \& Laughlin (2003) predict a final inclination distribution for dynamically relaxed planetary systems that peaks near $20^{\circ}$ and extends to inclinations as high as $85^{\circ}$. Kozai interactions between a planet and an outer body (star or planet) result in a wide distribution of final orbital inclinations for the inner planet, including retrograde orbits (Fabrycky \& Tremaine 2007; Wu et al. 2007; Nagasawa et al. 2008). Thus, measurements of both the orbital eccentricities and the orbital inclinations relative to the star's equator offer diagnostics of the predominant migration processes.

The Rossiter-McLaughlin (R-M) effect offers a way to assess quantitatively the spin-orbit alignment of a planetary system by measuring the Doppler effect of the star's light during a planetary transit. As the planet blocks a portion of a rotating star's surface receding from the observer, the spectrum from the unobscured surface has a net Doppler shift toward shorter wavelengths, and vice versa when it blocks a portion of the star approaching the observer. The R-M effect has been measured in 18 stars to date (Queloz et al. 2000; Winn et al. 2005, 2006, 2007b, 2008, 2009a; Wolf et al. 2007; Narita et al. 2007, 2008, 2009a, 2009b; Bouchy et al. 2008; Cochran et al. 2008; Johnson et al. 2008, 2009; Loeillet et al. 2008; Pont et al. 2009, 2010; Moutou et al. 2009; Simpson et al. 2010; Anderson et al. 2010; Gillon 2009; Triaud et al. 2009).

About two-thirds of the 18 planetary systems measured by the R-M effect have an orbital plane well aligned with the star's equatorial plane, as projected onto the sky, giving $\lambda$ near $0^{\circ}$. This alignment is as expected from simple migration theory due to gentle loss of orbital angular momentum to the gas in the protoplanetary disk (Lin et al. 1996). However, six exoplanetary systems show a significant spin-orbit misalignment, namely HD 80606 (Winn et al. 2009b; Moutou et al. 2009; Pont et al. 2010; Gillon 2009), WASP-14b (Johnson et al. 2009; Joshi et al. 2009), XO-3b (Hébrard et al. 2009; Winn et al. 2009c), HAT-P$7 \mathrm{~b}$ (Winn et al. 2009a; Narita et al. 2009a), CoRoT-1 (Pont et al. 2009), and Wasp-17b (Anderson et al. 2010).

The variety of alignments supports the bimodal distribution found by Fabrycky \& Winn (2009). Interestingly, five of the six misaligned systems contain quite massive planets, above $1 M_{\mathrm{J}}$, and the majority of well-aligned systems contain a planet with a mass less than $1 M_{\mathrm{J}}$. This correlation may be related to the association of massive planets with higher orbital eccentricity (Wright et al. 2009), as both eccentricity and inclination may arise from perturbations of planets from their original circular orbits. But there are two massive planets on eccentric orbits for which $\lambda$ appears to be consistent with zero, namely HD $17156 \mathrm{~b}$ (Cochran et al. 2008; Barbieri et al. 2009; Narita et al. 2009b) and HAT-P-2b (Winn et al. 2007a; Loeillet et al. 2008). There is currently no dominant and secure explanation for the misaligned or eccentric hot Jupiters.
Here, we present the first detection of the R-M effect from a planet detected by the Kepler Mission. As this mission is expected to detect dozens of transiting hot Jupiters, Kepler offers an opportunity to provide a statistically robust measure of the distribution of spin-orbit angles and to correlate that angle with other physical properties of the systems.

\section{KEPLER PHOTOMETRY}

Nearly continuous photometry in a $115 \mathrm{deg}^{2}$ field of view (FOV) near Cygnus and Lyra was carried out during 42 days by the Kepler space-borne telescope, as described previously (Borucki et al. 2010a, 2010b; Koch et al. 2010a; Jenkins et al. 2010a, 2010b; Batalha et al. 2010; Gautier et al. 2010). ${ }^{22}$ The star Kepler-8 (Kepler Input Catalog (KIC) $=6922244$, $\alpha=18^{\mathrm{h}} 45^{\mathrm{m}} 09.15, \delta=+42^{\circ} 27^{\prime} 03^{\prime \prime} .9$, J2000, KIC $r=$ $13.511 \mathrm{mag}$ ) exhibits a repeated dimming of $9.82 \pm 0.22 \mathrm{mmag}$, obvious against uncertainties in each 29.4 minute integration of $0.1 \mathrm{mmag}$. The light curve for Kepler- 8 is plotted in Figure 1 . The numerical data are available electronically at the MAST/ HLSP data archive. ${ }^{23}$ A modest amount of detrending has been applied (Koch et al. 2010b; Rowe et al. 2010) to the time series.

We detect no systematic difference between alternating transit events at $50 \mu$ mag levels, ruling out nearly equal components of an eclipsing binary star (see Figure 1). We also see no evidence of dimming at the expected times of a secondary eclipse, which would be visible for most eclipsing binary systems of unequal surface brightness. The photocenter shows no displacement astrometrically above mpix levels ( 0.5 mas) during times in and out of transit, as would be seen if there were a background eclipsing binary contaminating the image of the target star. Thus, the photometric and astrometric non-detections of a blended eclipsing binary support the planet interpretation for the repeated transit signatures. Moreover, the shape of the photometric transit is adequately fit with a planet-transit model, further supporting the planet interpretation.

We begin with a spectroscopic analysis assuming local thermodynamic equilibrium (LTE; Valenti \& Piskunov 1996; Valenti \& Fischer 2005) of a high-resolution template spectrum from Keck-HIRES of Kepler-8 to derive an effective temperature, $T_{\text {eff }}=6213 \pm 150 \mathrm{~K}$; surface gravity, $\log g=4.28 \pm 0.10$ (cgs); metallicity; $[\mathrm{Fe} / \mathrm{H}]=-0.055 \pm 0.03 ; v \sin i=10.5 \pm$ $0.7 \mathrm{~km} \mathrm{~s}^{-1}$; and the associated error distribution for each of them.

When sufficient radial velocity (RV) measurements had been obtained, we simultaneously fit the photometric and RV data. A $\chi^{2}$ minimization is performed to find the best-fitting solution. The analytic expressions of Mandel \& Agol (2002) are used to model the transit shape using Kepler-bandpass nonlinear limbdarkening parameters (Claret 2004). The data are fit for the center of transit time, period, impact parameter $(b)$, the scaled planetary radius $\left(R_{\mathrm{P}} / R_{\star}\right)$, the amplitude of the RV $(K)$, photometric and velocity zero points, and $\zeta / R_{\star}$. We also account for non-circular orbits by modeling for $e \cos (\omega), e \sin (\omega)$, where $e$ is the orbital eccentricity and $\omega$ is the argument of periastron. The term $\zeta / R_{\star}$ is related to the transit duration $\left(T_{d}=2\left(\zeta / R_{\star}\right)^{-1}\right)$ and the mean stellar density (Pál et al. 2010). The error

\footnotetext{
22 The first two data sets acquired by Kepler consist of $\sim 10$ days of data starting on 2009 May 3 and 35 days of data starting on 2009 May 12. These two data sets are called Q0 and Q1, respectively.

23 Time series of the photometry and of RV data presented here may be retrieved from the MAST/HLSP data archive at http://archive.stsci.edu/prepds/kepler_hlsp.
} 

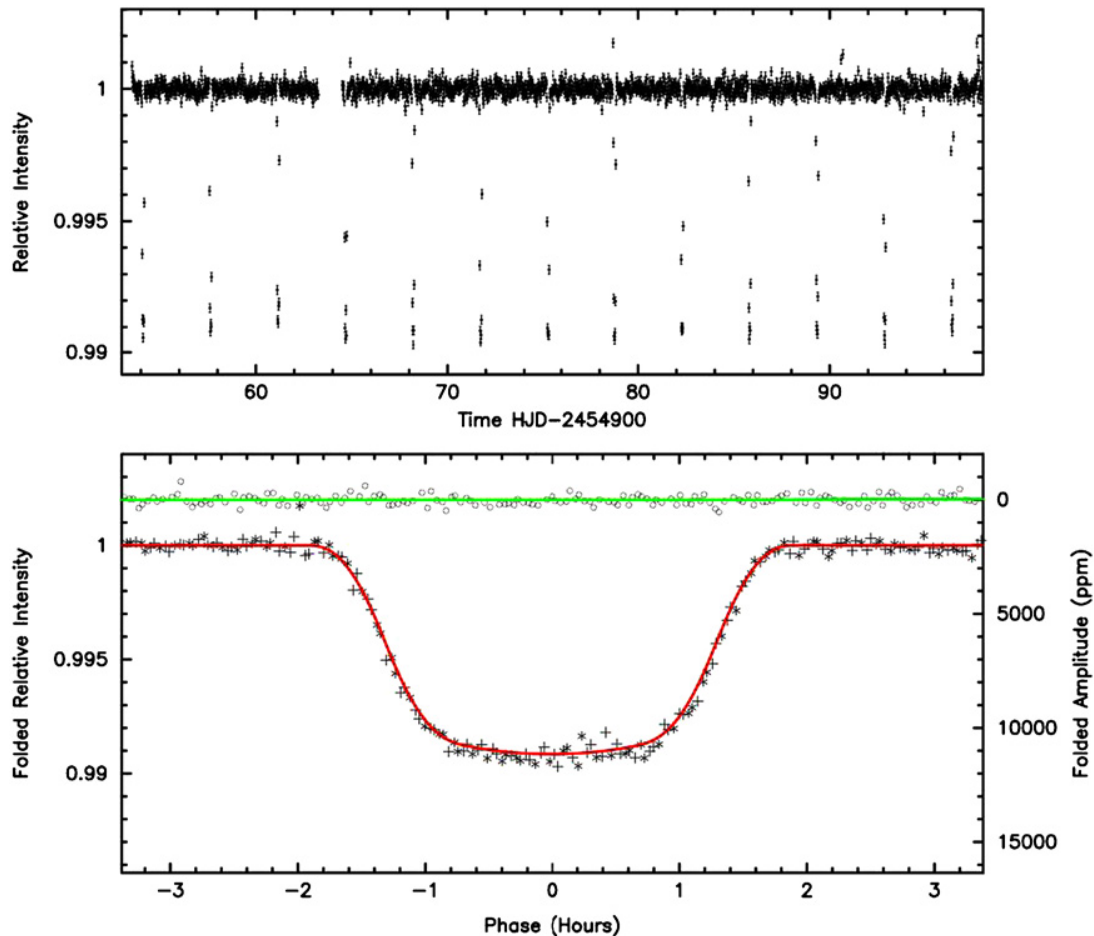

Figure 1. Detrended light curve for Kepler-8. The time series for the entire Kepler photometric data set is plotted in the upper panel. The lower panel shows the photometry folded by the period $P=3.52254$ days, and the model fit to the primary transit is plotted as a solid line. Data for even and for odd transits are denoted by "+"s and "*"s, respectively. The even and odd transits do not exhibit significant variations in transit depth indicative of the primary and secondary eclipses of an eclipsing binary. The photometry at the predicted time of occultation of the planet is shown just above the phased transit curve, showing no significant dimming at the level of $0.1 \mathrm{mmag}$. The lack of any dimming at the predicted time of occultation, along with the absence of both astrometric motion in and out of transit and the absence of detected stellar companions, leaves the planetary interpretation as the only plausible model, confirmed by the R-M detection.

(A color version of this figure is available in the online journal.)

distributions for the stellar densities that fit the transit light curve and account for variations in eccentricity are obtained from a Markov Chain Monte Carlo (MCMC) analysis (Ford 2005).

The multitudes of Yale-Yonsei stellar evolution models (Yi et al. 2004; Demarque et al. 2004) are constrained by both the LTE measurements and the stellar density that stems directly from the orbital period, the fractional dimming during transit, and measures of transit durations (Sozzetti et al. 2007; Brown 2010). By Monte Carlo analysis, those photometric and spectroscopic constraints and their uncertainties establish the probability density contours among the evolutionary tracks where the star may reside. We iterate the self-consistent fitting of light curves, RVs, and evolutionary models until a domain of stellar mass, radius, and age is identified. That domain encompasses a range of evolutionary states that satisfy all of the constraints within their error distributions.

The allowed $M_{\star}$ and $R_{\star}$ pairs are used to define a transition probability to compute an MCMC analysis to determine the most likely model values. We fit for stellar mass and radius, planetary mass and radius, center time of transit, orbital period and inclination, depth of the occultation, and $e \cos (\omega)$ and $e \sin (\omega)$ and reported in Table 1, along with other associated stellar properties such as luminosity and age.

\section{A BACKGROUND ECLIPSING BINARY: FOLLOW-UP IMAGING}

We carried out extensive tests of the possibility that the apparent photometric transit was actually caused by a background eclipsing binary star within the photometric aperture of radius $\sim 8^{\prime \prime}$. We obtained images with $0 ! 8$ seeing with the Keck telescope HIRES guider camera and the BG38 filter to search for stellar companions that might be eclipsing. The combination of this filter and the CCD detector has a response similar to the $V$ plus $R$ bands, similar to the bandpass of the Kepler photometer. This Keck image is shown in Figure 2. There is one star having 0.0075 of the flux of the main star ( $V$ plus $R$ band) that resides 3".8 northwest of Kepler-8. This background star resides within the Kepler aperture and could conceivably be an eclipsing binary that causes the combined light of the two stars to masquerade as a transiting planet.

But this faint neighboring star cannot be the source of the photometric transit signature for two reasons. If the background star were the cause of the observed $1 \%$ dimming, the photocenter centroid shifts would be 3-20 mpix on the Kepler CCD. Instead, astrometric measurements show shifts of no more than $\sim 0.1$ mpix for in-transit measurements relative to out-of-transit ones. Figure 3 shows both the flux and astrometric photocenter (centroid) of the Kepler images during Q1 of the data. We applied a high-pass filter to remove non-transit signatures on timescales longer than two days. At times of transits, there are no displacements in either the row or column direction at a level above $\sim 0.5$ mpix.

To hunt further for background eclipsing binaries, we plotted flux versus the astrometric photocenter centroids in both the row and column directions, as shown in Figure 4. These so-called rain plots would reveal an eclipsing binary as a displacement in the centroids to the left or right as the flux drops. No such displacement is detected at a level near 0.1 mpix (or 0.4 mas). This indicates that any background eclipsing binary would need 
Table 1

System Parameters for Kepler-8

\begin{tabular}{|c|c|c|}
\hline Parameter & Value & Notes \\
\hline \multicolumn{3}{|l|}{ Transit and orbital parameters } \\
\hline Orbital period, $P$ (days) & $3.52254_{-0.00005}^{+0.00003}$ & A \\
\hline Mid-transit time, $E$ (HJD) & $2454954.1182_{-0.0004}^{+0.0003}$ & A \\
\hline Scaled semimajor axis, $a / R_{\star}$ & $6.97_{-0.24}^{+0.20}$ & A \\
\hline Scaled planet radius, $R_{\mathrm{P}} / R_{\star}$ & $0.09809_{-0.00046}^{+0.00040}$ & A \\
\hline Impact parameter, $b \equiv a \cos i / R_{\star}$ & $0.724 \pm 0.020$ & A \\
\hline Orbital inclination, $i(\mathrm{deg})$ & $84.07 \pm 0.33$ & A \\
\hline Orbital semi-amplitude, $K\left(\mathrm{~m} \mathrm{~s}^{-1}\right)$ & $68.4 \pm 12.0$ & $\mathrm{~A}, \mathrm{~B}$ \\
\hline Orbital eccentricity, $e$ & 0 (adopted) & $\mathrm{A}, \mathrm{B}$ \\
\hline $\begin{array}{l}\text { Projected angle between planetary orbit and } \\
\text { stellar spin axis, } \lambda \text { (deg) }\end{array}$ & $-26.4 \pm 10.1$ & G \\
\hline \multicolumn{3}{|l|}{ Observed stellar parameters } \\
\hline Effective temperature, $T_{\text {eff }}(\mathrm{K})$ & $6213 \pm 150$ & $\mathrm{C}$ \\
\hline Spectroscopic gravity, $\log g(\mathrm{cgs})$ & $4.28 \pm 0.10$ & $\mathrm{C}$ \\
\hline Metallicity, $[\mathrm{Fe} / \mathrm{H}]$ & $-0.055 \pm 0.03$ & $\mathrm{C}$ \\
\hline Projected rotation velocity, $v \sin i\left(\mathrm{~km} \mathrm{~s}^{-1}\right)$ & $10.5 \pm 0.7$ & $\mathrm{C}$ \\
\hline Absolute (Helio) RV $\left(\mathrm{km} \mathrm{s}^{-1}\right)$ & $-52.72 \pm 0.10$ & $\mathrm{~B}$ \\
\hline \multicolumn{3}{|l|}{ Derived stellar parameters } \\
\hline Mass, $M_{\star}\left(M_{\odot}\right)$ & $1.213_{-0.063}^{+0.067}$ & A,C,D \\
\hline Radius, $R_{\star}\left(R_{\odot}\right)$ & $1.486_{-0.062}^{+0.053}$ & $\mathrm{~A}, \mathrm{C}, \mathrm{D}$ \\
\hline Surface gravity, $\log g_{\star}(\mathrm{cgs})$ & $4.174 \pm 0.026$ & $\mathrm{C}, \mathrm{D}$ \\
\hline Luminosity, $L_{\star}\left(L_{\odot}\right)$ & $4.03_{-0.54}^{+0.52}$ & $\mathrm{C}, \mathrm{D}$ \\
\hline Absolute $V$ magnitude, $M_{V}$ (mag) & $3.28 \pm 0.15$ & $\mathrm{D}$ \\
\hline Age (Gyr) & $3.84 \pm 1.5$ & $\mathrm{C}, \mathrm{D}$ \\
\hline Distance (pc) & $1330 \pm 180$ & $\mathrm{D}$ \\
\hline \multicolumn{3}{|l|}{ Planetary parameters } \\
\hline Mass, $M_{\mathrm{P}}\left(M_{\mathrm{J}}\right)$ & $0.603_{-0.19}^{+0.13}$ & $\mathrm{~A}, \mathrm{~B}, \mathrm{C}, \mathrm{D}$ \\
\hline Radius, $R_{\mathrm{P}}\left(R_{\mathrm{J}}\right)$ & $1.419_{-0.058}^{+0.056}$ & A,BC,D \\
\hline Density, $\rho_{\mathrm{P}}\left(\mathrm{g} \mathrm{cm}^{-3}\right)$ & $0.261 \pm 0.071$ & $\mathrm{~A}, \mathrm{~B}, \mathrm{C}, \mathrm{D}$ \\
\hline Surface gravity, $\log g_{P}$ (cgs) & $2.871 \pm 0.119$ & $\mathrm{~A}, \mathrm{~B}, \mathrm{E}$ \\
\hline Orbital semimajor axis, $a$ (AU) & $0.0483_{-0.0012}^{+0.0006}$ & $\mathrm{E}$ \\
\hline Equilibrium temperature, $T_{\mathrm{eq}}(\mathrm{K})$ & $1764 \pm 200$ & $\mathrm{~F}$ \\
\hline
\end{tabular}

Notes. A: based on the photometry; B: based on the RVs; C: based on an SME analysis of the Keck-HIRES spectra; D: based on the Yale-Yonsei stellar evolution tracks; E: based on Newton's version of Kepler's Third Law and total mass; F: assumes Bond albedo $=0.1$ and complete redistribution; G: based on RV observations of the R-M effect.

to be within $\sim 0.01$ pixels or 0 '.04 of Kepler-8 in order to explain the photometric transit signals (see Equation (A5)). Finally, we looked directly at the Kepler images taken both during and out of transit to detect motion of the centroid of light, as shown in Figure 5. We formed the difference of the images in and out of transit to detect astrometric displacements associated with the flux dimming, as would occur if a neighboring eclipsing binary were the cause. Those difference images show no shift of the photocenter. We conclude that the transit photometry with its $1 \%$ dimming cannot be explained by any eclipsing binary companions beyond $1^{\prime \prime}$ of Kepler- 8 .

Furthermore, the flux ratio of the two stars is only 0.0075 , making it impossible for the background star to cause the $1 \%$ photometric dimming. Even if that background star were to vanish, the total flux would decline by less than the observed $1 \%$. We conclude that the $1 \%$ photometric dimming is not caused by an eclipsing binary star within the Kepler photometric aperture, from $\sim 0.1^{\prime \prime}$ to $10^{\prime \prime}$ of the target star.

To hunt for additional stars in the field within an aresec of Kepler-8, we used both speckle imaging and adaptive optics (AO) imaging. Kepler- 8 was observed at the Palomar Hale

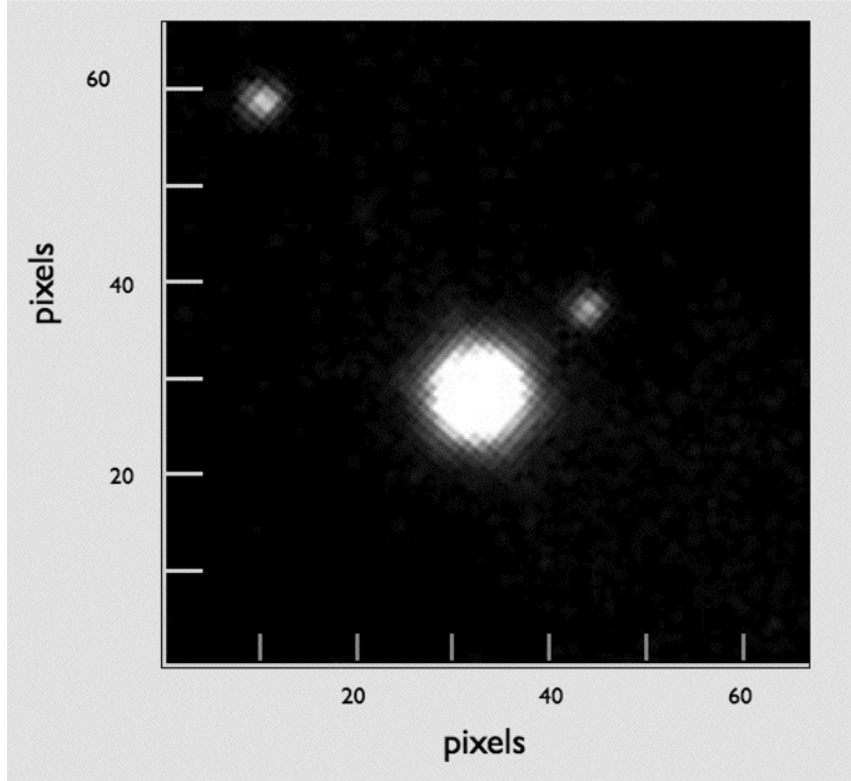

Figure 2. Image of the field near Kepler-8 obtained from the Keck 1 telescope guider camera with a BG38 filter, making the bandpass approximately $V$ and $R$ combined, similar to that of the Kepler photometer. North is up, east to the left, and the plate scale is 0.397 pixel $^{-1}$. The main star, Kepler-8, is the brightest source at the center of the image. A faint star is located $3 . .8$ to the northwest of Kepler- 8 , having a flux $0.75 \%$ that of Kepler- 8 and residing within the Kepler photometric aperture. With a flux less than $1 \%$ that of Kepler- 8 , the companion cannot be responsible for the periodic $1 \%$ dimming, even if it were a background eclipsing binary. But this neighboring star, if constant, is sufficiently bright to account for the astrometric displacement of Kepler-8 that occurs during transit of its planet, explaining the shift of the photocenter of the two stars by 0.0001 Kepler pixels.

200 inch telescope on 2009 September 9 UT with the Palomar near-infrared AO system (PHARO; Hayward et al. 2001). The PHARO instrument was utilized in the $J$-band filter with the 25 mas pixel $^{-1}\left(25^{\prime \prime}\right.$ FOV $)$ mode. The source was observed with a five-point dither and an integration time of $2.8 \mathrm{~s}$ per frame. The dither pattern was repeated seven times for a total onsource integration time of $98 \mathrm{~s}$. The average uncorrected seeing during the observations at $J$ band was $00^{\prime} 65$, and the average AO-corrected images produced point-spread functions (PSFs) that were 0 '.09 full width at half-maximum (FWHM). There are two faint sources within $4^{\prime \prime}$ of Kepler-8; these objects are 7 mag and 8.4 mag fainter than the primary target and are too faint to produce the Kepler-observed transit and centroid shift. The AO imaging detected no sources at $J$ band down to within $0{ }^{\prime \prime} 1-0.2$ of the primary target that are within $\Delta m \approx 6-7$ mag at $J$.

Speckle observations of Kepler- 8 were made on 2009 October 1 UT at the WIYN observatory located on Kitt Peak. The observations were made using the WIYN speckle camera during a night of very good seeing $(0,62)$ and under clear conditions. We used a narrow-bandpass, $40 \mathrm{~nm}$ wide filter centered at $692 \mathrm{~nm}$. The Kepler speckle program obtains observations of both double and single standard stars throughout the night. We use a robust background estimator on the reconstructed images to set a limit for the level of companion star we should detect if present. The speckle observations show that Kepler- 8 has no companion star between 0 '.05 and 2'. 0 within a delta magnitude of $<4.2$ mag. These AO and speckle observations effectively rule out the possibility of an eclipsing binary star to within as close as 0 .' 1 from Kepler-8. 

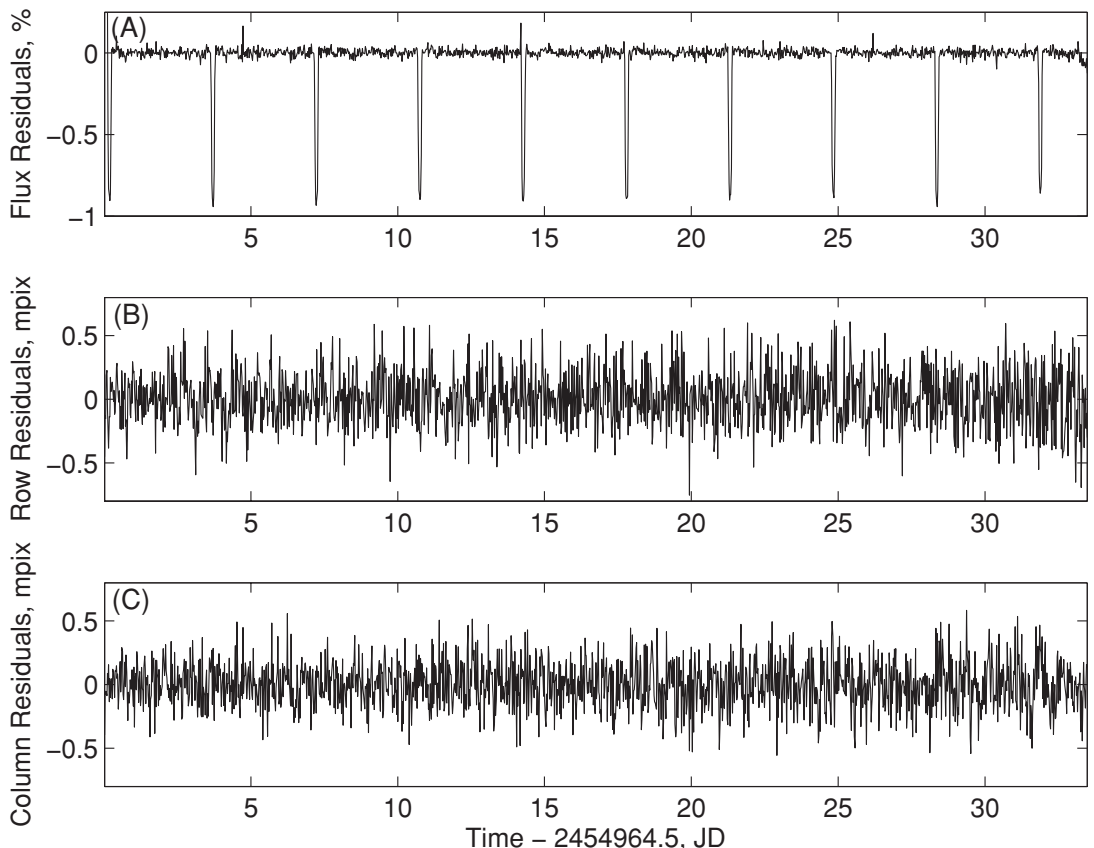

Figure 3. Flux (A) and astrometric centroid row (B) and column (C) time series of Kepler-8b from Kepler Q1 data. A high-pass filter has been applied to each time series to remove non-transit signatures on timescales longer than two days. There is no obvious displacement of the centroid of light in either the row or column residuals. This lack of motion indicates that any background eclipsing binary would need to be located within $\sim 0.001 / 0.01=0.1$ pixels, corresponding to 0 "' 4 of the target star, in order to explain the photometric transit signals but avoid showing motion.

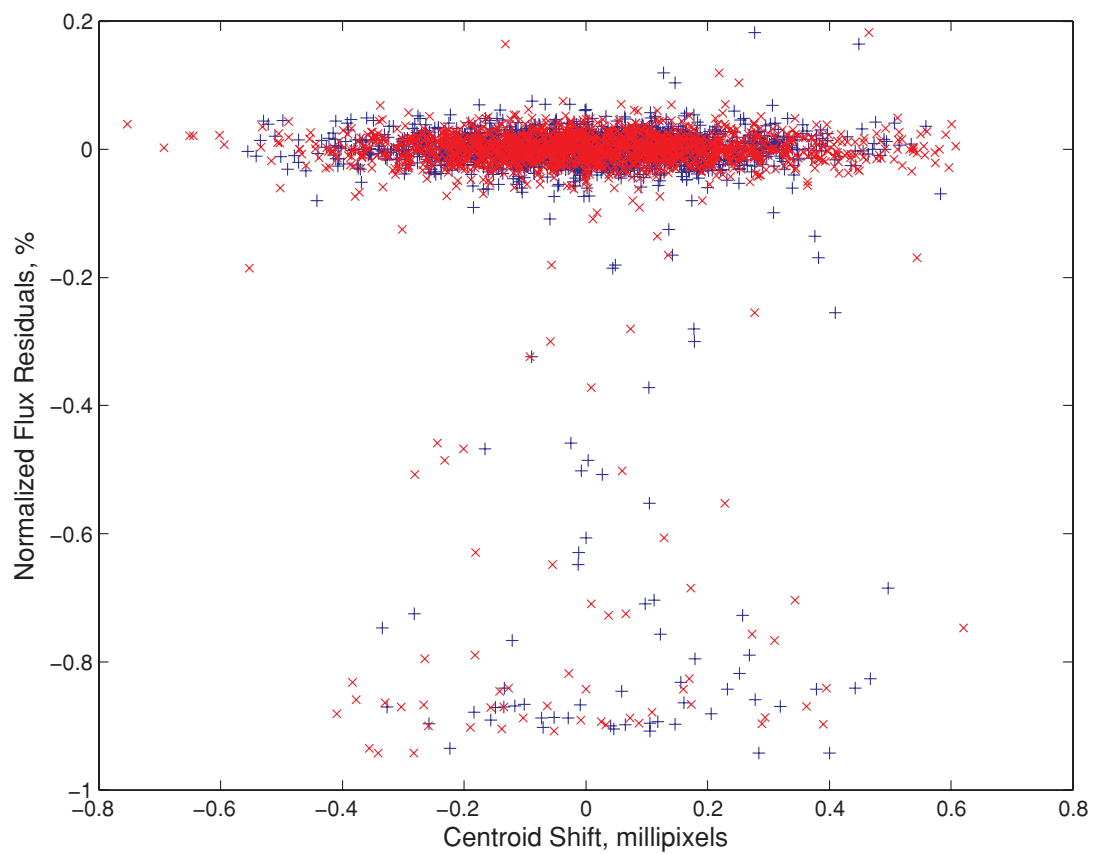

Figure 4. Flux vs. residual astrometric centroids in row $(x)$ and column $(+)$ for Kepler-8. The measurements are the same as those presented in Figure 3. This "rainplot" shows no evidence that the centroids are systematically shifting during each transit event, which would be indicative of a blend with a background star. If this were the case, identification of the actual star responsible for the transit features in the light curve would require inspection of a high-resolution image or catalog to unravel the mystery. Since the in-transit points "rain" straight down, rather than slanting along a diagonal, any blend scenario would require a very small separation on the sky between the two sources.

(A color version of this figure is available in the online journal.)

\subsection{Covariance of Inclination, Limb Darkening, and Stellar Parameters}

As Kepler-8b has a large impact parameter, the solution is quite sensitive to errors in limb darkening. The derived impact parameter and inclination angle are directly related to the assumed limb-darkening law, which is poorly known for the wide
Kepler bandpass. We adopted an ad hoc approach to estimate the limb-darkening parameters (Rowe et al. 2010) as follows. We noted that fits to the observed R-M effect (see Section 6), coupled with the measured $v \sin i=10.5 \pm 0.7 \mathrm{~km} \mathrm{~s}^{-1}$, offered a constraint on the inclination angle and the impact parameter, and hence on the inferred stellar radius, for a given limb-darkening law. Alternatively, one may compare the 
(A)

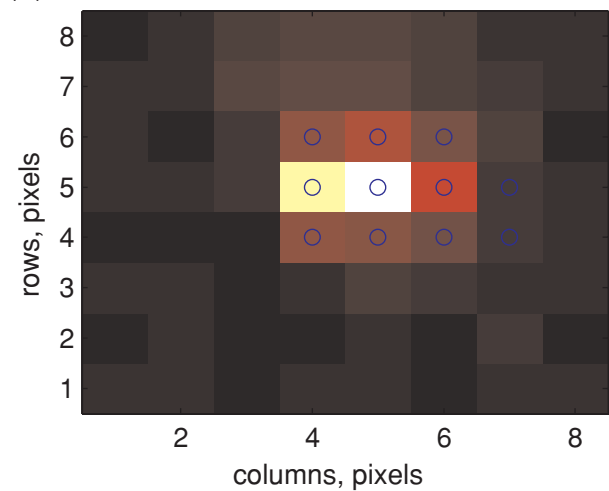

(C)

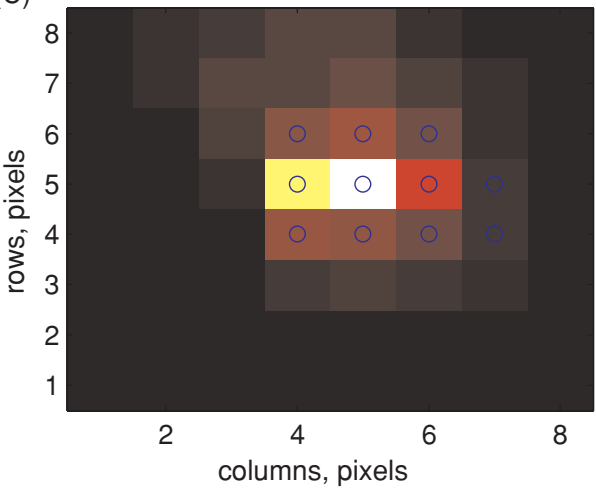

(B)
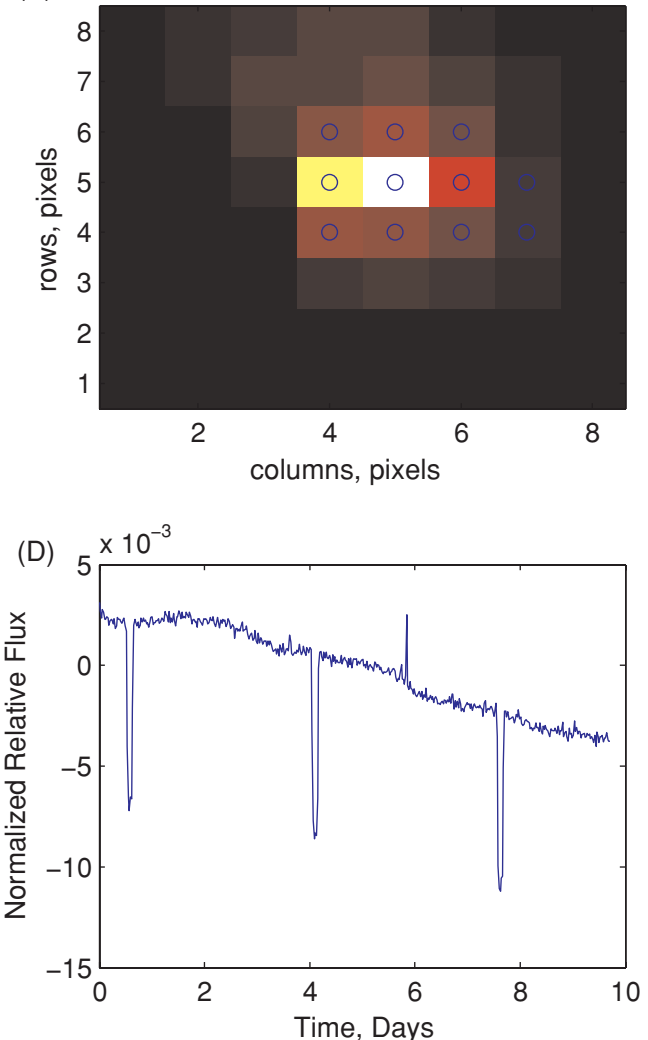

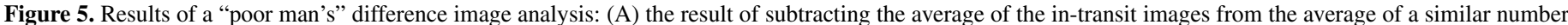

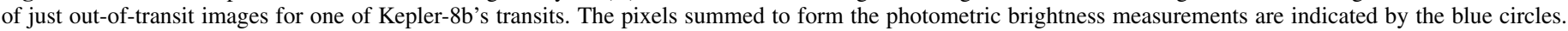

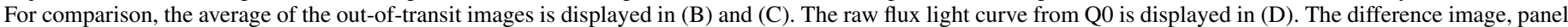

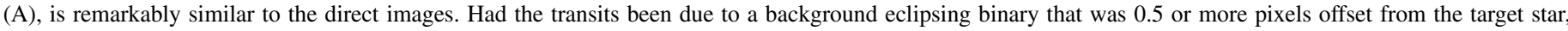

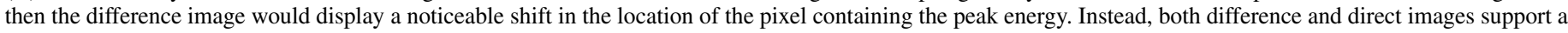
coincident localization of the source of the transits to the target star, Kepler-8.

(A color version of this figure is available in the online journal.)

values of $v \sin i$ from Spectroscopy Made Easy (SME; Valenti \& Piskunov 1996; Valenti \& Fischer 2005) to that coming from the R-M analysis. Such analyses offered evidence of an inconsistency in the parameters. Solving the general case for eccentric orbits (Pal et al. 2010) did not resolve the inconsistency.

We pursued this inconsistency in parameters offered by the R-M measurement. For the cases of Kepler-4 and Kepler-5 (Borucki et al. 2010b; Koch et al. 2010b), we discovered that a model using tabular values (Prsa \& Zwitter 2006) for limbdarkening overpredicted the amount of curvature in the variation of flux as a function of time during transit. We therefore modified the limb-darkening parameters by fitting the three previously known exoplanets in the Kepler field, TRES-2, HAT-P-7, and HAT-P-11, and using published values for their stellar and planetary parameters. We linearly interpolated over the values of effective temperature to derive a superior measure of the true limb-darkening law for the Kepler bandpass. The photometry from new transiting planets found by Kepler, along with IR photometry that is less sensitive to limb darkening, will allow us to refine the limb-darkening law for the Kepler bandpass in the coming months. The resulting fits to the photometric transits of Kepler-4, 5, 6, 7, and 8 were all improved with our new limbdarkening treatment (Rowe et al. 2010).

For Kepler-8b, the resulting impact parameter and inclination were $b=0.724 \pm 0.020$ and $i=84.07 \pm 0.33$, both about $10 \%$ smaller than we had obtained by using the first-guess limbdarkening law. The transit duration in turn dictates the best-fit

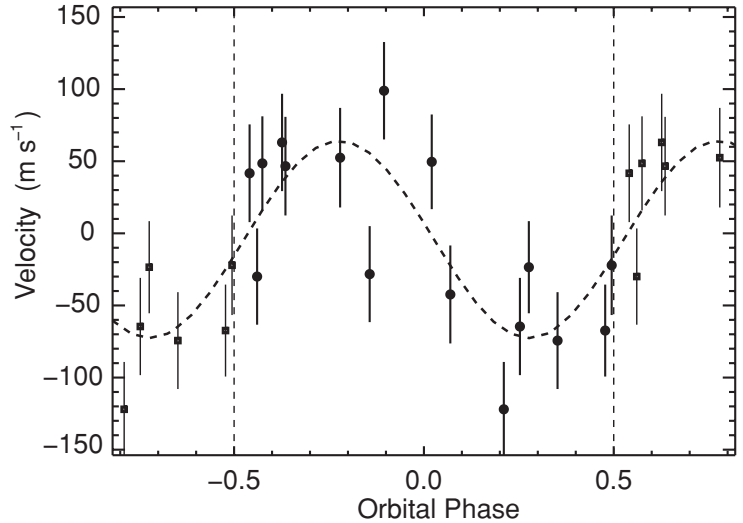

Figure 6. Orbital solution for Kepler-8. The observed RVs obtained with HIRES on the Keck 1 telescope are plotted together with the best-fit velocity curve for a circular orbit with the period and time of transit fixed by the photometric ephemeris. Uncertainties are large due to the faintness of the star $(V=13.9 \mathrm{mag})$, the high rotational Doppler broadening ( $v \sin i=10.5 \pm 0.7$ ), and contamination from background moonlight in most spectra. The measured velocities vary high and low as predicted by the orbit from the photometric transit curve, supporting the planet model.

stellar radius, and after iterating with the Yale-Yonsei models, the resulting stellar radius is $R=1.486_{-0.062}^{+0.053} R_{\odot}$. The stellar mass has a value within a narrow range, $M=1.213_{-0.063}^{+0.067} M_{\odot}$, again derived from iteration between the fit to the light curve and to the Yale-Yonsei models. Additional information on 
Table 2

Relative Doppler Velocity Measurements of Kepler- 8

\begin{tabular}{|c|c|c|}
\hline $\begin{array}{c}\text { HJD } \\
(-2450000) \\
\end{array}$ & $\begin{array}{c}\mathrm{RV} \\
\left(\mathrm{m} \mathrm{s}^{-1}\right)\end{array}$ & $\begin{array}{c}\sigma_{\mathrm{RV}} \\
\left(\mathrm{m} \mathrm{s}^{-1}\right)\end{array}$ \\
\hline 4984.040029 & -22.15 & 34.5 \\
\hline 4985.042856 & 52.45 & 34.5 \\
\hline 4986.065428 & -42.39 & 34.0 \\
\hline 4987.060244 & -74.37 & 33.6 \\
\hline 4988.026564 & 63.04 & 33.7 \\
\hline 4988.972744 & 98.87 & 33.8 \\
\hline 4995.103097 & 46.58 & 34.2 \\
\hline 5014.891326 & -64.61 & 33.7 \\
\hline 5015.975326 & -29.97 & 33.4 \\
\hline 5017.019858 & -28.28 & 33.3 \\
\hline 5075.788330 & 41.65 & 33.9 \\
\hline 5109.849781 & -122.00 & 32.8 \\
\hline 5110.790309 & -67.42 & 31.9 \\
\hline 5133.717887 & 58.11 & 32.6 \\
\hline 5133.732343 & 51.13 & 32.5 \\
\hline 5133.747053 & 71.85 & 32.7 \\
\hline 5133.761161 & 34.51 & 32.8 \\
\hline 5133.775640 & 24.38 & 32.6 \\
\hline 5133.790026 & -9.49 & 33.2 \\
\hline 5133.804527 & -31.04 & 33.2 \\
\hline 5133.821205 & -14.32 & 32.6 \\
\hline 5133.838751 & 49.58 & 32.8 \\
\hline 5134.739907 & -23.48 & 31.9 \\
\hline 5135.789679 & 48.50 & 32.6 \\
\hline
\end{tabular}

stellar mass comes from the projected rapid rotation of the star, $v \sin i=10.5 \pm 0.7 \mathrm{~km} \mathrm{~s}^{-1}$. This $v \sin i$ is faster than typical rotation for stars at the lower end of the mass range quoted above, especially as some evolution and associated increase in moment of inertia has occurred. Thus, we marginally favor the upper half of the quoted mass range, i.e., $M>1.213 M_{\odot}$. The stellar gravity is $\log g=4.174 \pm 0.026$ (cgs), and the approximate age is $3.84 \pm 1.5 \mathrm{Gyr}$, both indicating a star nearing the end of its main-sequence lifetime.

\section{RADIAL VELOCITIES}

We took high-resolution spectra of Kepler-8 using HIRES on the Keck I 10 m telescope (Vogt et al. 1994). We set up the HIRES spectrometer in the same manner that has been used consistently for 10 years with the California planet search (Marcy et al. 2008). We employed the red cross-disperser and used the $\mathrm{I}_{2}$ absorption cell to measure the instrumental profile and the wavelength scale. The slit width was set at 0.87 by the "B5 decker," giving a resolving power of about 60,000 at $5500 \AA$. Between 2009 June 1 and 2009 October 31, we gathered 15 spectra of Kepler-8 out of transit (see Figure 6). Typical exposure times were between 10 and 45 minutes, yielding signal-to-noise ratios (S/Ns) of 20-40 pixel $^{-1}$. These are quite low S/Ns with which to attempt precise Doppler measurements. We tested the Doppler precision from such low $\mathrm{S} / \mathrm{N}$ spectra on standard stars, notably HD 182488 and HD 9407, and found that the expected errors are $\sim 5 \mathrm{~m} \mathrm{~s}^{-1}$, as expected from Poisson statistics of the photons, with no systematic errors above $1 \mathrm{~m} \mathrm{~s}^{-1}$, as usual for the iodine technique. On 2009 October 29 (UT), we obtained nine spectra of Kepler-8 during transit (the last taken during egress). The observations were taken between airmass 1.9 and 3.8, while the star was setting, the last exposure occurring at hour angle $5 \mathrm{hr}$ 35 minutes with extreme atmospheric refraction and dispersion. Again, tests with standard stars observed at such high airmass show no systematic errors in the Doppler measurements above $1 \mathrm{~m} \mathrm{~s}^{-1}$.

We carried out careful reduction of the raw images, including cosmic ray elimination and optimal extraction of the spectra, to minimize the background moonlight. We performed the Doppler analysis with the algorithm of Johnson et al. (2009). We estimated the measurement error in the Doppler shift derived from a given spectrum based on the weighted standard deviation of the mean among the solutions for individual $2 \AA$ spectral segments. The typical internal measurement error was $7-10 \mathrm{~m} \mathrm{~s}^{-1}$. The resulting velocities are given in Table 2 and plotted in Figure 6.

The actual uncertainties in the velocities are certainly closer to $20-25 \mathrm{~m} \mathrm{~s}^{-1}$ for several reasons. The spectra have such low photon levels, $\sim 200$ photons per pixel in the raw CCD images, that cosmic rays and background sky are significant noise sources. The latter was especially problematic as we used an entrance slit with dimensions 0 .' $87 \times 3$ 3.5 not long enough to separate the wings of the star's PSF from the background sky. Nearly all HIRES spectra were taken with the moon gibbous or full, and about half of the nights had moderate cirrus that scatters moonlight into the slit. A few observations made with a $14^{\prime \prime}$ slit revealed that $1 \%-3 \%$ of the light came from the moonlit sky in typical observations. Simulations with stellar spectra contaminated by moonlit sky suggested that errors of $\sim 10 \mathrm{~m} \mathrm{~s}^{-1}$ would accrue, no doubt systematic as well. Errors in the velocities are large because of relatively rapid rotation, $v \sin i=10.5 \pm 0.7$, broadening the absorption lines by $5 \times$ that of the slowest rotating stars. The uncertainty in Doppler shift is proportional to the line width and inversely proportional to the square root of the number of pixels within the lines. Hence, the Doppler shift uncertainty increases as the square root of the line width. Thus, while the $\mathrm{S} / \mathrm{N}=40 \mathrm{might}$ be expected to yield velocities of $\sim 5 \mathrm{~m} \mathrm{~s}^{-1}$ for a slowly rotating star, the high $v \sin i$ increases that error to $\sim 20 \mathrm{~m} \mathrm{~s}^{-1}$. Indeed, the best orbital fit to the velocities, shown in Figure 6, exhibits discrepancies of $20-30 \mathrm{~m} \mathrm{~s}^{-1}$. We estimate that the true errors in velocities are thus $\sim 25 \mathrm{~m} \mathrm{~s}^{-1}$. We have accounted for these errors by adding a "jitter" of $25 \mathrm{~m} \mathrm{~s}^{-1}$ to the internal velocity errors. Those augmented uncertainties are reflected in Table 2.

We carried out a Levenberg-Marquardt least-squares fit of a Keplerian single-planet model to the observed velocities. In all models, the orbital period and the time of mid-transit were constrained to be that found in the photometric fit. We first assumed a circular orbit leaving only two free parameters in our fit, namely the velocity amplitude and the gamma velocity of the system. The best-fit model is overplotted in Figure 6. The best-fit value of the amplitude is $K=68.4 \pm 12.0 \mathrm{~m} \mathrm{~s}^{-1}$. This, coupled with the adopted stellar mass of $1.213_{-0.063}^{+0.067} M_{\odot}$, yields a planet mass of $0.603_{-0.19}^{+0.13} M_{\mathrm{J}}$.

We also carried out fits in which the eccentricity was allowed to float. The best-fit model has an eccentricity $e=0.24$, $K=71.4 \mathrm{~m} \mathrm{~s}^{-1}$, yielding a planet mass of $0.62 M_{\mathrm{J}}$. The rms of the residuals is $39 \mathrm{~m} \mathrm{~s}^{-1}$ compared with $40 \mathrm{~m} \mathrm{~s}^{-1}$ for the circular orbit, rendering the eccentric orbit no better than the circular. Indeed, a bootstrap Monte Carlo estimate of the parameter uncertainties gives a formal error in eccentricity of 0.16 . We conclude that the eccentricity is consistent with zero, but could be as high as 0.4 . This large uncertainty stems from the high $v \sin i$ of the star and its relative faintness, $V=13.9 \mathrm{mag}$. We note that the previous work by Lucy \& Sweeney (1971), Shen \& Turner (2008), and O'Toole et al. (2009) also found that Keplerian fits to low S/N RV observations are biased away from zero eccentricity. 
A line bisector analysis showed no variation at a level of $\sim 12 \mathrm{~m} \mathrm{~s}^{-1}$ and no correlation with measured RVs. Thus, the velocity variation appears to represent actual acceleration of the center of mass of the star.

\section{PROPERTIES OF THE PLANET KEPLER-8b}

With the planet interpretation highly likely, the properties of the host star and the depth of transit directly yield a planet radius of $1.419_{-0.058}^{+0.056} R_{\mathrm{J}}$ and a mass of $0.603_{-0.19}^{+0.13} M_{\mathrm{J}}$. The errors represent the $68 \%$ probability domain of integrated uncertainty from all input measurements and models. The planet density is $0.261 \pm 0.071 \mathrm{~g} \mathrm{~cm}^{-3}$, placing it among the lowdensity exoplanets. An equilibrium temperature for the planet of $1764 \pm 200 \mathrm{~K}$ is obtained by using the best-fit stellar luminosity from the Yale-Yonsei models, assuming a Bond albedo of 0.1 and that redistribution of thermal energy around the planet's surface is both rapid and complete. This planet apparently is a member of the population of low density, bloated hot Jupiters having thermal histories yet to be firmly understood. The planet properties are listed in Table 1.

\section{ROSSITER-MCLAUGHLIN EFFECT}

The RVs obtained during the transit of 2009 October 29 (UT) were modeled using the techniques described by Cochran et al. (2008). This method tiles the disk of the star with a grid of cells and then integrates over Doppler-shifted and intensity-weighted stellar photospheric cells that are not blocked by the transiting planet to compute the R-M RV perturbation during the transit. In essence, we numerically integrate Equation (20) of Ohta et al. (2005), rather than use the analytic formulae they developed. As was pointed out by Winn et al. (2005), the fundamental assumption behind the R-M effect observations, i.e., that a distorted line profile shape may be analyzed as a Dopplershifted line profile, is not strictly true. Essentially, all analysis techniques that do not exactly mimic the observational process will break down with increasing $v \sin i$ and with increasing stellar line width. Hirano et al. (2010) developed an additional correction term to the Ohta et al. (2005) analytic formulae, which includes a term dependent on the stellar line width as well as a $v \sin i$-dependent term.

Our models adopted the system parameters in Table 1 and searched for the value of $\lambda$ that produced the best match to the observed velocities. In particular, we assumed a zeroeccentricity orbit. We used the same four-parameter, quadratic limb-darkening law as was used for the transit light curve analysis, although running similar models with either a linear or a Claret (2004) quadratic limb-darkening law appropriate to this star gave very similar results. We derive a value of $\lambda=$ $-26.4 \pm 4.6$ for the angle between the projected stellar rotation axis and the projected planetary orbital axis. The model fit to the data gives a reduced chi-squared $\chi_{0}^{2}=0.66$ for the velocities obtained during the transit, indicating that the uncertainties in these velocities are probably slightly overestimated. We note that the R-M model fit to the data has just a single free parameter, $\lambda$. All of the other model parameters were fixed to the values obtained either from the light curve analysis or from the RV orbital solution.

The RVs measured during the transit and the R-M effect model fit are shown in Figure 7. The observed asymmetric R-M effect, with a positive deviation of significantly larger amplitude and longer duration than the negative amplitude, is a result of the combination of the large impact parameter and the non-

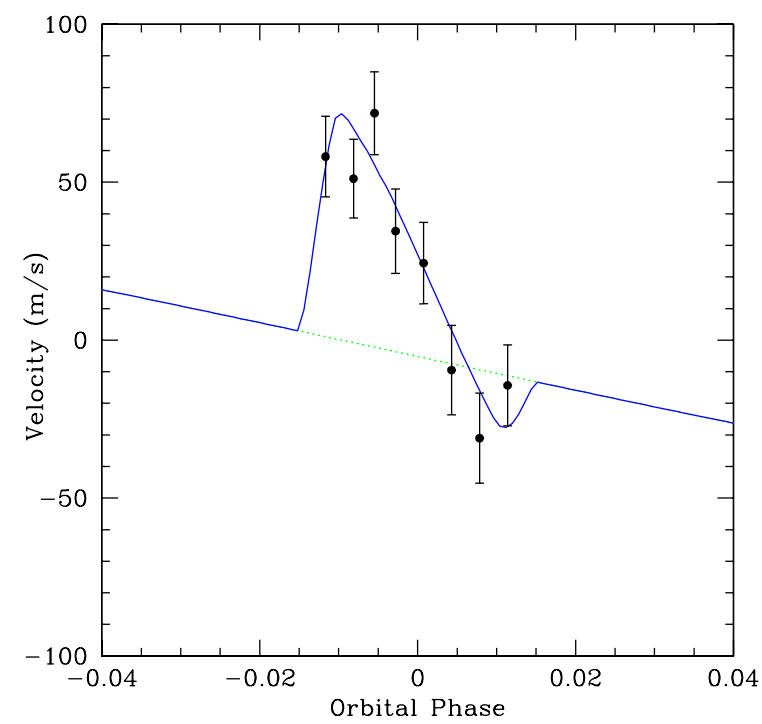

Figure 7. R-M effect for Kepler-8. The observed RVs obtained with HIRES on the Keck 1 telescope are plotted together with the predicted velocities from the best-fit model of the R-M effect. The high velocities near ingress and low velocities near egress imply a prograde orbit. The asymmetry in the model curve indicates a projected tilt of the orbit relative to the star's equator, as well as a transit with a large impact parameter on the star. The errors presented here in the RVs measured during transit are smaller than the errors in the other RV measurements due to the fact that a long slit was used, allowing for better sky subtraction, and also because the RV jitter was smaller during the $3 \mathrm{hr}$ of the transit.

(A color version of this figure is available in the online journal.)

zero value of $\lambda$. A central transit would give a symmetric R-M deviation, with the amplitude of the effect then depending on $\lambda$. However, for an off-center transit, the overall shape depends critically on both the impact parameter and $\lambda$ (cf. Figure 2 of Gaudi \& Winn 2007). Note that the RV measurements made during the transit were smaller than the prior out-of-transit measurements due to the fact that a longer slit was used, allowing for better sky subtraction, and also because the RV jitter was smaller during the $3 \mathrm{hr}$ transit.

The duration of the RV observations during transit on 2009 October 29 was limited by evening twilight and by the setting of the star. Thus, observations started shortly after ingress and ended during egress. No velocities outside of transit could be obtained that night. The measured velocities during the transit show a decreasing trend with time. The evidence for the nonzero value of $\lambda$ comes from the excess of positive over negative velocity deviations, as shown in Figure 7. Indeed, if all of these velocities were shifted downward by about $30 \mathrm{~m} \mathrm{~s}^{-1}$, a symmetric R-M effect with $\lambda \sim 0^{\circ}$ would result. Therefore, we investigated very carefully whether these velocities obtained during the transit may have been subject to any sort of systematic error that might have shifted them uniformly upward, resulting in the observed R-M velocity perturbation. Velocities for these observations were computed in exactly the same manner as for all of the out-of-transit observations. There is nothing in the observation or data reduction process that we know of that could cause these velocities to have a different zero point than any of the other velocities. We see no evidence for systematic velocity offsets from any of the other nights of Keck observations. One possible astrophysical source of a velocity offset would be starspots. If there were a large starspot blocking part of the blueshifted stellar hemisphere, this could conceivably cause a net apparent redshift to all of the velocities obtained during this night. However, the Kepler light curve shows no evidence of 
features consistent with large starspots or with significant spot activity. Moreover, the Keck HIRES spectra include the region of the $\mathrm{Ca}$ II $\mathrm{H}$ and $\mathrm{K}$ lines, which are good indicators of stellar activity. Velocity jitter of Kepler-8 might result from magnetic activity and surface motions typical for an F8 main-sequence star with $v \sin i=10.5 \mathrm{~km} \mathrm{~s}^{-1}$. Examination of the $\mathrm{Ca}$ II H and $\mathrm{K}$ lines shows no significant emission cores, indicating a weak chromosphere. We measured the chromospheric emission at $\mathrm{Ca}$ II $\mathrm{H}$ and $\mathrm{K}$ in the three HIRES spectra having the highest $\mathrm{S} / \mathrm{N}$. The average of those measures gave $S=0.173$ and $\log R_{\mathrm{HK}}^{\prime}=-4.916$, showing that Kepler-8 has low magnetic activity. Such stars typically show a jitter of $5 \mathrm{~m} \mathrm{~s}^{-1}$ with a timescale of many hours, arguing against the possibility of such an offset during the R-M measurements.

Nevertheless, in order to understand the possible effects of any unknown systematic errors on our R-M analysis, we computed a set of models with the observed in-transit velocities shifted by $\pm 10 \mathrm{~m} \mathrm{~s}^{-1}$. If all of the transit velocities are increased by $10 \mathrm{~m} \mathrm{~s}^{-1}$, we derive $\lambda=-35.6 \pm 4.6$, and if the transit velocities are decreased by $10 \mathrm{~m} \mathrm{~s}^{-1}, \lambda=-17.7 \pm 4.5$ results. Thus, a $10 \mathrm{~m} \mathrm{~s}^{-1}$ systematic velocity offset results in about a $-9^{\circ}$ change in $\lambda$. This $10 \mathrm{~m} \mathrm{~s}^{-1}$ offset is significantly larger than any possible systematic error that we can imagine. Nevertheless, to be conservative, we include this level of possible systematic error in the uncertainties of $\lambda$ to obtain a final value for Kepler- $8 \mathrm{~b}$ of $\lambda=-26.4 \pm 10.1$.

This R-M analysis provides independent verification of the major results of the transit light curve analysis. The light curve analysis alone suggested a transit chord that is significantly off center, with impact parameter $b=0.724 \pm 0.020$ and inclination $i=84.07 \pm 0.33$. This large impact parameter coupled with the non-zero value of $\lambda$ results in the planet-transit blocking mostly the approaching (blueshifted) half of the rotating star, as indicated in Figure 8. The planet crosses to the receding (redshifted) portion of the stellar disk just before the end of the transit. This causes the observed asymmetry in the R-M velocity perturbation during the transit. The observed amplitude of the $\mathrm{R}-\mathrm{M}$ effect is in excellent agreement with the photometrically determined stellar radius and impact parameter and with the spectroscopically determined $v \sin i$. The duration of the photometric transit agrees with the duration of the observed R-M velocity perturbation. This consistency between the properties of the system as derived from the transit photometry and from the R-M velocity perturbation gives a confirmation that both phenomena were caused by an orbiting, planet-sized companion to the star. There is no other explanation of the observed $\mathrm{R}-\mathrm{M}$ variations that is also consistent with both the photometric and the RV observations.

This observation of the R-M effect in Kepler-8 is important because it supports the suggestion that the observed light curve and RV variations were indeed caused by an orbiting planetary companion. Alternatively, a background eclipsing binary system with the same 3.5 day period would be tidally coupled, resulting in rotational velocities of $\sim 20 \mathrm{~km} \mathrm{~s}^{-1}$. Eclipsing a portion of such a rotating stellar surface would cause spectral distortions located $\sim 20 \mathrm{~km} \mathrm{~s}^{-1}$ from line center, larger than the line widths of Kepler- 8 and greater than the few $\mathrm{m} \mathrm{s}^{-1}$ effect observed here. The detection of a smooth R-M effect here rules out such typical eclipsing binaries. Moreover, background eclipsing binaries consisting of an early-type primary are immediately ruled out by our detection of the R-M effect since A and B stars have so few lines. Thus, our detection of the R-M effect adds support to the existence of the planet, which was already quite

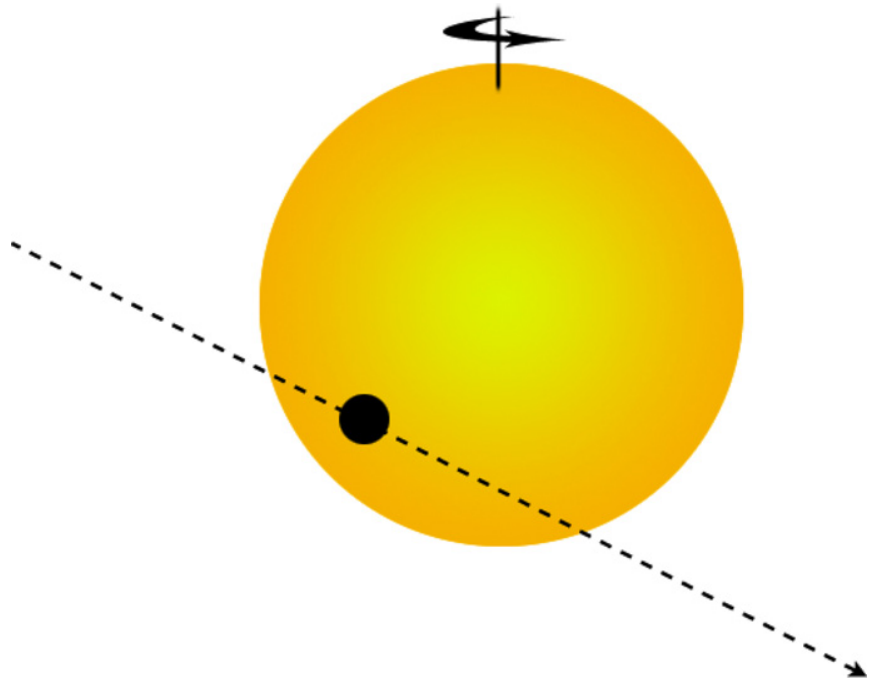

Figure 8. Sketch of the transit geometry for Kepler-8. The Kepler photometry and R-M measurements set the impact parameter, $b=0.724 \pm 0.020$, and the angle projected on the sky between the star's spin axis and the normal to the orbital plane, $\lambda=-27^{\circ}$, indicating a prograde orbit with a moderate inclination. (A color version of this figure is available in the online journal.)

compelling from the photometric, astrometric, imaging, and RV observations.

\section{DISCUSSION}

We have carried out a wide array of observations of Kepler- 8 that pinpoint the existence and properties of the exoplanet. The Kepler photometric measurements, with $\sim 0.1 \mathrm{mmag}$ errors in 30 minute intervals, are tightly fit by a model of a transiting exoplanet (Figure 1). This good fit to the inflections of the photometric data provides immediate support for the planet interpretation, with few plausible alternative interpretations except for a background, diluted eclipsing binary system having just the right brightness and radius ratio to mimic a planet. We note that, unlike ground-based transit work that has lower photometric precision, the Kepler photometry is so precise that blends of eclipsing binaries are more readily identified from the photometry alone.

Nonetheless, to test the unlikely possibility of a blend, we carried out a battery of astrometric tests to hunt for an eclipsing binary. The resulting steadiness of the position of the primary star, Kepler-8, during and out of transit, argues against any eclipsing binary in the photometric aperture. We further carried out both $\mathrm{AO}$ imaging and speckle interferometric measurements to hunt for faint eclipsing binaries located within an arcsec of the Kepler-8. None was found, further diminishing the chance of such a masquerade. We followed with high-resolution spectroscopy at both low and high $\mathrm{S} / \mathrm{N}$, finding no evidence of double lines nor rapid rotation. Further support for the planet interpretation came from the precise RVs that varied in phase with, and had the same period as, the photometric light curve, further supporting the planet model and constraining the planet mass.

Finally, the R-M effect confirmed independently the planet interpretation and provided further geometrical information about the orbit, notably $\lambda=-26.4 \pm 10.1$. A sketch of the star and planet's orbit is shown in Figure 8. Remaining unknown is the inclination of the star's rotation axis, but continued photometry during the Kepler Mission lifetime may reveal a photometric periodicity caused by the rotation of spots around 
the star. The resulting rotation period, coupled with the measured rotational $v \sin i=10.5 \pm 0.7$ of the star, will allow the star's inclination to be measured, putting the R-M geometry on firmer ground.

The suite of ground-based observations obtained for Kepler- 8 described above overconstrained a set of parameters that were obligated to mutually agree, including such subtle issues as the limb darkening, the stellar rotation rate, and stellar radius, mass, and density. Thus, the R-M effect can, in general, be used to confirm the existence of a planetary companion in the case where standard follow-up observing procedures may provide somewhat ambiguous or equivocal results. Examples would be Kepler stars that are sufficiently saturated that measurement of photocenter shifts during transits is problematic, or stars like Kepler- 8 with large $v \sin i$ that make orbit determination via precise RV measurement difficult.

Observations of the R-M effect in dozens of transiting planets, discovered from the ground and from Kepler, offer an excellent opportunity to determine the distribution of orbital geometries of the short-period planetary systems in general. These planetary systems will continue to be studied in an extraordinarily uniform and consistent manner with the same set of tools. The sample selection effects are extremely well understood and documented. The Kepler light curves are of unprecedented precision, allowing sensitive searches for additional planets in the system via transit timing variations and significantly tighter limits to be placed in the future on the orbital eccentricity. Coupling these data with $\mathrm{R}-\mathrm{M}$ measurements of the orbital alignment in these systems will allow us to correlate the observed properties with the degree of orbital alignment and thus to search for the physical mechanisms causing observed misalignments. We will also have the information to be able to begin to back out the physical angle between the spin and orbital angular momentum vectors, not just the projection of these angles on the plane of the sky.

Funding for this mission is provided by NASA's Science Mission Directorate. Many people have contributed to the success of the Kepler Mission, and it is impossible to acknowledge them all. Valuable advice and assistance were provided by Willie Torres, Riley Duren, M. Crane, D. Ciardi, and Josh Winn. Special technical help was provided by Carly Chubak, G. Mandushev, and Josh Winn. We thank E. Bachtel and his team at Ball Aerospace for their work on the Kepler photometer and R. Thompson for key contributions to engineering, and C. Botosh for able management. G.W.M. thanks and acknowledges support from NASA Cooperative Agreement NNX06AH52G.

Facility: Kepler.

\section{APPENDIX}

\section{DERIVATION OF CENTROID SHIFT CORRELATIONS FOR MULTIPLE SOURCES}

One of the most important sources of false positives for transit surveys is the population of background eclipsing binaries. Analysis of the motion of the photocenter or centroid of the target star can detect correlations between the transitlike features in the photometric time series and shifts in the photocenter indicating a blend situation. The interpretation of the centroid shift to identify which star is the source of the transit-like photometric signature can be complicated for crowded fields, but is tractable in most cases. Here, we derive the effect of an eclipsing background binary on the centroid of a blended target star and examine the efficacy with which we can detect the induced centroid shift and ascertain the nature of its source.

Consider a collection of point sources with fluxes $b_{i}$ and locations $\left\{x_{i}, y_{i}\right\}$ for $i=0, \ldots, m-1$. Let us define the total brightness of the system of sources as $B=\sum_{i=0}^{m-1} b_{i}$. The centroid $\mathbf{x}_{c}=\left\{x_{c}, y_{c}\right\}$ of the system of point sources is given by

$$
\mathbf{x}_{c}=\frac{\sum_{i} \mathbf{x}_{i} b_{i}}{\sum_{i} b_{i}} .
$$

If the brightness of the system changes by an amount $\delta b$ due to source $k \in\{0, \ldots, m-1\}$, then the fractional change in brightness is $\delta b / B$, and the centroid of the system will be perturbed by

$$
\delta \mathbf{x}_{k}=\left\{\delta x_{k}, \delta y_{k}\right\}=\frac{\sum_{i} \mathbf{x}_{i} b_{i}+\delta b \mathbf{x}_{k}}{\sum_{i} b_{i}+\delta b}-\frac{\sum_{i} \mathbf{x}_{i} b_{i}}{\sum_{i} b_{i}}
$$

in each axis.

A Taylor series expansion of Equation (A2) about the term $\delta b / B$ gives the following:

$$
\begin{aligned}
\delta \mathbf{x}_{k}= & \left.\frac{\sum_{i} \mathbf{x}_{i} b_{i}+\mathbf{x}_{k} \delta b}{\sum_{i} b_{i}+\delta b}\right|_{\delta b=0}-\frac{\sum_{i} \mathbf{x}_{i} b_{i}}{\sum_{i} b_{i}}+\left.\frac{d}{d \delta b}\left\{\frac{\sum_{i} \mathbf{x}_{i} b_{i}+\mathbf{x}_{k} \delta b}{\sum_{i} b_{i}+\delta b}\right\}\right|_{\delta b=0} \delta b \\
& +\left.\frac{1}{2} \frac{d^{2}}{d \delta b^{2}}\left\{\frac{\sum_{i} \mathbf{x}_{i} b_{i}+\mathbf{x}_{k} \delta b}{\sum_{i} b_{i}+\delta b}\right\}\right|_{\delta b=0} \delta b^{2}+\bigcirc\left(\delta b^{3}\right) \\
= & \frac{\sum_{i} \mathbf{x}_{i} b_{i}}{B}-\frac{\sum_{i} \mathbf{x}_{i} b_{i}}{B}+\left.\frac{(B+\delta b) \mathbf{x}_{k}-\left(\sum_{i} \mathbf{x}_{i} b_{i}+\mathbf{x}_{k} \delta b\right)}{(B+\delta b)^{2}}\right|_{\delta b=0} \delta b \\
& +\left.\frac{1}{2} \frac{(B+\delta b)^{2}\left(\mathbf{x}_{k}-\mathbf{x}_{k}\right)-\left(B \mathbf{x}_{k}-\sum_{i} \mathbf{x}_{i} b_{i}\right) 2(B+\delta b)}{(B+\delta b)^{4}}\right|_{\delta b=0} \delta b^{2}+\bigcirc\left(\delta b^{3}\right) \\
= & \frac{\mathbf{x}_{k} B-\sum_{i} \mathbf{x}_{i} b_{i}}{B^{2}} \delta b-\frac{\mathbf{x}_{k} B^{2}-B \sum_{i} \mathbf{x}_{i} b_{i}}{B^{4}} \delta b^{2}+\bigcirc\left(\delta b^{3}\right) \\
= & \left(\mathbf{x}_{k}-\mathbf{x}_{c}\right) \frac{\delta b}{B}-\left(\mathbf{x}_{k}-\mathbf{x}_{c}\right)\left(\frac{\delta b}{B}\right)^{2}+\bigcirc\left[\left(\frac{\delta b}{B}\right)^{3}\right] .
\end{aligned}
$$

For planetary transits, $\delta b / B \ll 1$, so that Equation (A3) reduces to

$$
\delta \mathbf{x}_{k}=\Delta \mathbf{x}_{k} \frac{\delta b}{B},
$$

where $\Delta \mathbf{x}_{k}=\mathbf{x}_{k}-\mathbf{x}_{c}$ is the offset between source $k$ and the mean out-of-transit centroid of the collection of sources in the aperture, and the term $\delta b / B$ is simply the depth of the "transits" detected in the first place. Thus, during each transit-like event the centroid will move away from the true source of the photometric signature by the distance between the true source and the mean out-of-transit centroid diluted by the fractional "transit depth." The offset of the true source location from the mean out-oftransit location can be estimated as

$$
\Delta \mathbf{x}=\delta \mathbf{x}\left(\frac{\delta b}{B}\right)^{-1}
$$

where the subscript $k$ has been omitted since the number of sources is not known in general, and the two terms on the righthand side of the equation are the observables.

Assuming that the observation noise is Gaussian, the uncertainty in the estimate of the source offset, $\sigma_{\Delta \mathbf{x}}^{2}$, can be obtained as

$$
\sigma_{\Delta \mathbf{x}}^{2}=(\delta b / B)^{-2}\left[\sigma_{\delta \mathbf{x}}^{2}+\delta \mathbf{x}^{2} \sigma_{\delta b / B}^{2}\right],
$$

where $\sigma_{\delta \mathbf{x}}^{2}$ and $\sigma_{\delta b / B}^{2}$ are the uncertainties in the centroid measurement and the transit depth, respectively. 
Additional insight can be gained by considering the case restricted to two stellar sources: the $\operatorname{target} \operatorname{star}(k=0)$ and a dim background star $(k=1)$ with $B \approx b_{0} \gg b_{1}$. Without loss of generality, we can set $x_{0}=0$ so that $x_{1}=\Delta x$ is the separation between the two stars. Under the hypothesis that the target star is the source of the transit-like brightness variations, Equation (A4) reduces to

$$
\begin{aligned}
\delta \mathbf{x}_{0} & =\left(\mathbf{x}_{0}-\mathbf{x}_{c}\right) \frac{\delta b}{B}=\left(\mathbf{x}_{0}-\frac{\mathbf{x}_{0} b_{0}+\mathbf{x}_{1} b_{1}}{b_{0}+b_{1}} \frac{\delta b}{B}\right) \approx-\mathbf{x}_{1} \frac{b_{1}}{B} \frac{\delta b}{B} \\
& =-\Delta \mathbf{x} \frac{b_{1}}{B} \frac{\delta b}{B},
\end{aligned}
$$

while under the hypothesis that the background star is the source, we obtain

$$
\begin{aligned}
\delta \mathbf{x}_{1} & =\left(\mathbf{x}_{1}-\mathbf{x}_{c}\right) \frac{\delta b}{B}=\frac{\mathbf{x}_{1} b_{0}+\mathbf{x}_{1} b_{1}-\mathbf{x}_{0} b_{0}-\mathbf{x}_{1} b_{1}}{B} \frac{\delta b}{B} \\
& \approx \mathbf{x}_{1} \frac{\delta b}{B}=\Delta \mathbf{x} \frac{\delta b}{B} .
\end{aligned}
$$

Note that $\delta \mathbf{x}_{0} / \delta \mathbf{x}_{1}=b_{1} / B$, the ratio of the brightness of the background star to that of the target star. For the case of most interest to Kepler, namely that of an Earth-size transit signature $\left(\delta b / B=-1 \times 10^{-4}\right)$, and a background star $9.3 \mathrm{mag}$ fainter, the centroid shift will be 5000 times greater for the case of a background eclipsing binary than for the case of a transiting planet orbiting the brighter target star. In general, then, background eclipsing binaries exert much greater leverage on the centroid of a simple blend than does the brighter target star, making it more likely that a significant centroid shift will be caused by a background eclipsing binary for small transit signatures.

When can we detect a significant centroid shift? A centroid shift statistic can be formed by fitting the row and column centroid time series against the modeled transit signature, squaring the fitted amplitudes in each axis, normalizing by the uncertainty in each fit, and summing the normalized components:

$$
T_{\delta \mathbf{x}}=\frac{\delta x^{2}}{\sigma_{\delta x}^{2}}+\frac{\delta y^{2}}{\sigma_{\delta y}^{2}} .
$$

$T_{\delta \mathbf{x}}$ is a $\chi^{2}$ variable with 2 degrees of freedom (assuming Gaussian statistics) with the probability distribution $F_{\chi_{2}^{2}}(x)=$ $\gamma(1, x / 2)$, where $\gamma$ is the incomplete Gamma function. The significance of a measured centroid shift during transit can then be assessed against an appropriate false rejection rate.

For the canonical case of an eclipsing binary mimicking an Earth-size transit of a $K p=12 \mathrm{G} 2 \mathrm{~V}$ star in the Kepler starfield, what is the confusion radius, that distance within which we cannot reliably detect centroid motion due to a background eclipsing binary? The centroiding precision over a $6.5 \mathrm{hr}$ interval for this star is given approximately by the ratio of the FWHM of the PSF ( 1.2 pixels; Van Cleve \& Caldwell 2009) to the $\mathrm{S} / \mathrm{N}$ of the flux measurement (Monet et al. 2010). A $K p=12 \mathrm{G} 2 \mathrm{~V}$ star provides $\sim 5 \times 10^{9} e^{-}$in $6.5 \mathrm{hr}$, so that $\sigma_{\delta x} \approx 8 \mu$ pix for a set of four transits. A background eclipsing binary $9.247 \mathrm{mag}$ fainter exhibiting full $(50 \%)$ eclipses that is closer than 0.25 pixels or $1^{\prime \prime}$ will introduce a centroid shift that is less than three times the centroiding precision, marking the outer radius for detection.

The results developed in this appendix apply in the strict sense only to infinitesimal PSFs. However, for the case of close blends with separations $<1$ pixel (or $4^{\prime \prime}$ ) from the target star, the results presented here are valid. Caution should be exercised when using Equation (A5) to estimate the location of the source of a transit-like signature to account for cases where the transit signature source is widely offset from the target star. Difference images such as presented in Figure 5 are valuable in identifying cases where the eclipsing binary is on the edge or outside of the photometric aperture.

\section{REFERENCES}

Adams, F. C., \& Laughlin, G. 2003, Icarus, 163, 290

Anderson, D. R., et al. 2010, ApJ, 709, 159

Barbieri, M., et al. 2009, A\&A, 503, 601

Batalha, N. M., et al. 2010, ApJ, 713, L103

Borucki, W. J., et al. 2010a, Science, 325, 709

Borucki, W. J., et al. 2010b, ApJ, 713, L126

Bouchy, F., et al. 2008, A\&A, 482, L25

Brown, T. M. 2010, ApJ, 709, 535

Chatterjee, S., Ford, E. B., Matsumura, S., \& Rasio, F. A. 2008, ApJ, 686, 580 Claret, A. 2004, A\&A, 428, 1001

Cochran, W. D., Redfield, S., Endl, M., \& Cochran, A. L. 2008, ApJ, 683, L59

Demarque, P., Woo, J.-H., Kim, Y.-C., \& Yi, S. K. 2004, ApJS, 155, 667

Fabrycky, D., \& Tremaine, S. 2007, ApJ, 669, 1298

Fabrycky, D. C., \& Winn, J. N. 2009, ApJ, 696, 1230

Ford, E. B. 2005, ApJ, 129, 1706

Gaudi, B. S., \& Winn, J. N. 2007, ApJ, 655, 550

Gautier, T. N., III, et al. 2010, arXiv:1001.0352

Gillon, M. 2009, arXiv:0906.4904

Hayward, T. L., Brandl, B., Pirger, B., Blacken, C., Gull, G. E., Schoenwald, J., \& Houck, J. R. 2001, PASP, 113, 105

Hébrard, G., et al. 2009, IAU Symp. 253, Transiting Planets, ed. F. Point, D. Sasselov, \& M. Holman (Cambridge: Cambridge Univ. Press), 508

Hirano, T., Suto, Y., Taruya, A., Narita, N., Sato, B., Johnson, J. A., \& Winn, J. N. 2010, ApJ, 709, 458

Holman, M., Touma, J., \& Tremaine, S. 1997, Nature, 386, 254

Jenkins, J. M., et al. 2010a, ApJ, 713, L87

Jenkins, J. M., et al. 2010b, ApJ, 713, L120

Johnson, J. A., Winn, J. N., Albrecht, S., Howard, A. W., Marcy, G. W., \& Gazak, J. Z. 2009, PASP, 121, 1104

Johnson, J. A., et al. 2008, ApJ, 686, 649

Joshi, Y. C., et al. 2009, MNRAS, 392, 1532

Koch, D. G., et al. 2010a, ApJ, 713, L79

Koch, D. G., et al. 2010b, ApJ, 713, L131

Lin, D. N. C., Bodenheimer, P., \& Richardson, D. C. 1996, Nature, 380 606

Loeillet, B., et al. 2008, A\&A, 481, 529

Lucy, L. B., \& Sweeney, M. A. 1971, AJ, 76, 544

Mandel, K., \& Agol, E. 2002, ApJ, 580, L171

Marcy, G. W., et al. 2008, Phys. Scr., 130, 014001

Monet, D. G., Jenkins, J. M., Dunham, E. W., Bryson, S. T., Gilliland, R. L., Latham, D. W., Borucki, W. J., \& Koch, D. G. 2010, arXiv:1001.0305

Moorhead, A. V., \& Adams, F. C. 2008, Icarus, 193, 475

Moutou, C., et al. 2009, A\&A, 498, L5

Nagasawa, M., Ida, S., \& Bessho, T. 2008, ApJ, 678, 498

Narita, N., Sato, B., Hirano, T., \& Tamura, M. 2009a, PASJ, 61, L35

Narita, N., Sato, B., Ohshima, O., \& Winn, J. N. 2008, PASJ, 60, L1

Narita, N., et al. 2007, PASJ, 59, 763

Narita, N., et al. 2009b, PASJ, 61, 991

Ohta, Y., Taruya, A., \& Suto, Y. 2005, ApJ, 622, 1118

O’Toole, S. J., Tinney, C. G., Jones, H. R. A., Butler, R. P., Marcy, G. W., Carter,

B., \& Bailey, J. 2009, MNRAS, 392, 641

Pál, A., et al. 2010, MNRAS, 401, 2665

Papaloizou, J. C. B., \& Terquem, C. 2001, MNRAS, 325, 221

Pont, F., et al. 2009, A\&A, 502, 695

Pont, F., et al. 2010, MNRAS, 402, L1

Prša, A., \& Zwitter, T. 2006, Ap\&SS, 304, 347

Queloz, D., Eggenberger, A., Mayor, M., Perrier, C., Beuzit, J. L., Naef, D.,

Sivan, J. P., \& Udry, S. 2000, A\&A, 359, L13

Rasio, F. A., \& Ford, E. B. 1996, Science, 274, 954

Rowe, J. F., et al. 2010, ApJ, 713, L150

Shen, Y., \& Turner, E. L. 2008, ApJ, 685, 553

Simpson, E. K., et al. 2010, MNRAS, 405, 1867

Sozzetti, A., Torres, G., Charbonneau, D., Latham, D. W., Holman, M. J., Winn, J. N., Laird, J. B., \& O’Donovan, F. T. 2007, ApJ, 664, 1190 
Torres, G., Winn, J. N., \& Holman, M. J. 2008, ApJ, 677, 1324

Triaud, A. H. M. J., et al. 2009, A\&A, 506, 377

Valenti, J. A., \& Fischer, D. A. 2005, ApJS, 159, 141

Valenti, J. A., \& Piskunov, N. 1996, A\&AS, 118, 595

Van Cleve, J., \& Caldwell, D. A. 2009, in Kepler Instrument Handbook, KSCI 19033-001 (Moffett Field, CA: NASA Ames Research Center), http://archive.stsci.edu/kepler

Vogt, S. S., et al. 1994, Proc. SPIE, 2198, 362

Ward, W. R., \& Hahn, J. M. 1994, Icarus, 110, 95

Winn, J. N., Holman, M. J., \& Fuentes, C. I. 2007a, AJ, 133, 11

Winn, J. N., Johnson, J. A., Albrecht, S., Howard, A. W., Marcy, G. W., Crossfield, I. J., \& Holman, M. J. 2009a, ApJ, 703, L99
Winn, J. N., et al. 2005, ApJ, 631, 1215

Winn, J. N., et al. 2006, ApJ, 653, L69

Winn, J. N., et al. 2007b, ApJ, 665, L167

Winn, J. N., et al. 2008, ApJ, 682, 1283

Winn, J. N., et al. 2009b, ApJ, 703, 2091

Winn, J. N., et al. 2009c, ApJ, 700, 302

Wolf, A. S., Laughlin, G., Henry, G. W., Fischer, D. A., Marcy, G., Butler, P., \& Vogt, S. 2007, ApJ, 667, 549

Wright, J. T., Upadhyay, S., Marcy, G. W., Fischer, D. A., Ford, E. B., \& Johnson, J. A. 2009, ApJ, 693, 1084

Wu, Y., Murray, N. W., \& Ramsahai, J. M. 2007, ApJ, 670, 820

Yi, S. K., Demarque, P., \& Kim, Y.-C. 2004, Ap\&SS, 291, 261 